\title{
Neuroinvasive potential of a primary respiratory pathogen SARS- CoV2: Exploring the underrecognized
}

\author{
Durjoy Lahiri ${ }^{1}$, Ritwick Mondal ${ }^{1}$, Shramana $_{\text {Deb }}^{2}$, Deebya Bandyopadhyay ${ }^{1}$,Gourav shome ${ }^{3}$ \\ Sukanya Sarkar ${ }^{4}$, Subhas c Biswas ${ }^{4}$ \\ 1- Institute of Post Graduate Medical Education and Research, SSKM Hospital, Kolkata, India. \\ 2- S.N Pradhan centre for Neuroscience, University of Calcutta, India \\ 3- Department of Microbiology, University of Calcutta, India. \\ 4-CSIR-Indian Institute of Chemical Biology, Kolkata, India
}

\begin{abstract}
After the emergence of Severe Acute Respiratory Syndrome Coronavirus(SARS-CoV) and Middle East Respiratory Syndrome Coronavirus (MERS-CoV) in the last two decades, the world is facing its new challenge in SARS-CoV-2 pandemic with unfathomable global responses. The characteristic clinical symptoms for Coronavirus (COVID-19) affected patients are high fever, drycough, dyspnoea, lethal pneumonia whereas some patients also show few additional neurological signs such as headache, nausea, vomiting. The accumulative evidences suggest that Coronavirus is not only confined within the respiratory tract and that may also invade in central nervous system (CNS), peripheral nervous system (PNS) inducing some fatal Neurological diseases. Here we analyse the phylogenetic perspective of SARS-CoV2 with other strains of $\beta$-Coronaviridae from a standpoint of neurological spectrum disorders. Based on the existing case reports, literature and open data-bases, we also analyse the differential distribution of neurological impairments in COVID-19 positive patients along with angiotensin-converting enzyme-2(ACE2) expression dynamics in neuronal and nonneuronal tissue of central and peripheral nervous system. Besides, we discuss the need for modulations in clinical approach from a neurological point of view, as a measure towards reducing disease transmission, morbidity and mortality in SARS-CoV2 positive patients.
\end{abstract}

Key words- COVID-19, ACE2, SARS-CoV, Neurological Disorders, Phylogenetic perspective, Coronavirus, CNS, PNS 


\section{Introduction}

The recent worldwide outbreak was caused by a novel virus named Wuhan coronavirus or 2019 novel coronavirus (2019-nCoV) by the Chinese researchers and later it was named as Severe-AcuteRespiratory-Syndrome-2 (SARS-CoV2) by International Committee on Taxonomy of Viruses (ICTV) [1].SARS-CoV2, first reported in Wuhan city, Hubei province of China since December 2019 and soon spread nationwide and rapidly spilled all over the world. Currently, it has spread to 203 countries/territories worldwide, with $>2,096,549$ infected cases and $>1,35,661$ casualties as of current date and increasing (www.worldometers.info/coronavirus/).

Coronaviruses belong to the Coronaviridae family in the Nidovirales order. The subgroups of this single stranded RNA virus include alpha, beta, gamma, delta and SARS-CoV-2 was reported to be a member of Beta group. Genetic analysis revealed that SARS-CoV-2 is phylogenetically related with SARS-CoV and MERS-CoV, which caused two other outbreaks in the year 2003 in China, Guangdong province and in 2012 in Saudi Arabia respectively[1].SARS-CoV-2 shares a significant amount of homology with SARS-CoV like bat viruses which suggest that bat could be a key reservoir. The human to human transfer is confirmed based on the world wide data but the intermediate host is still unknown.[1] The first case report in Huanan Seafood Wholesale market of Wuhan suggests that initially SARS-CoV-2 showed animal-to-human transmission but subsequent cases confirmed about human-to-human transmission and symptomatic patients are the most frequent source of COVID-19 spread[2].

\section{1- Initial attachment, entry and clinical features of SARS-CoV2}

The initial attachment of the virion particles to the host cell starts with interactions between the spike (S) protein with the host's receptor. The various sites of receptor binding domains (RBD) within the $\mathrm{S} 1$ region of a coronavirus $\mathrm{S}$ protein varies depending upon the virus itself, with some having the RBD at the C-terminus of S1 (SARS-CoV, SARS-CoV-2) while others (Murine Hepatitis Virus strain-1/MHV-1) have the RBD at the N-terminus of S1 $[\mathbf{3 , 4}]$. The S-protein-receptor interaction is 
the primary interaction which determines how a coronavirus can infect a host species and also governs the tissue tropism of the virus. Many coronaviruses utilize peptidases as their cellular receptor. It is unclear why peptidases are used, as entry occurs even in the absence of the enzymatic domain of these proteins [5]. Many $\alpha$-coronaviruses utilize aminopeptidase-N (APN) as their receptor. SARS-CoV, HCoV-NL63 and SARS-COV2 use angiotensin-converting enzyme 2 (ACE2) as their receptor, MHV enters through CEACAM1 (Carcinoembyonic Antigen-Related Cell Adhesions Molecules), MERSCoV binds to dipeptidyl-peptidase 4 (DPP4) to gain entry into human cells.[6]

The next major step may be the replication and transcription of the viral genome after endocytosis into the cell. Many set of genes that help during replication like the non-structural protein genes nsp1nsp16 are then transcribed inside the host cell [7]. Following translation of protein, assembly takes place within major cellular vesicles, utilized for assembly of structural proteins such as nucleoprotein, membrane protein, spike protein (N, M, S respectively). Virions are released afterwards[8]. Accumulative evidences suggest that the incubation time could be generally within 3-7 days and extend up to 2 weeks [2].The characteristic clinical symptoms for the COVID-19 affected patients are high fever, dry cough, dyspnoea, lethal pneumonia whereas some patients show some additional neurological signs such as headache, nausea and vomiting.

\section{2-The Phylogenetic homology and its correlation with the possibilities of developing neurological impairments in SARS-CoV-2 infected patients}

The genome sequences of all the reported SARS-CoV-2 share a similarity of around 99\% or more. (NCBI database) Sequencing studies followed with Genome Wide Association Studies (GWAS), suggest that the strain primarily isolated in Wuhan, china has a spectacular similarity with the bat derived coronavirus namely bat-SL-CoVZC45 and bat-SL-CoVZXC21[9]. In a recent phylogenetic network Analysis study of 160 human SARS-CoV2 genomes, three major central variants have been traced, named: A, B and C; as per their dissimilarities in amino acid sequence with type-A being the ancestral type. Phylogeographic patterns show, type A and C in considerable proportion outside East Asia, mainly in Europeans and Americans; while type B remains the most common in East Asia and its ancestral genome somehow shows resilience not to have spread outside this Area without mutating 
into derived B types, inclining towards founder effects or immunological or environmental resistance outside East Asia.[10]

On the other hand, the virus shows an identity match on the Blast platform with the SARSn-Cov (79\%) and distantly with the MERS-CoV (50\%). All of them fall under the family of betacoronavirus. Although it was genetically distinct from the SARS-CoV and more similar to the bat counterpart of the coronavirus. Homology modelling based studies have shown an identical receptor binding site with similar domain structure irrespective of having key differences in the amino acid sequence between SARS-CoV and n-COV 2019 with ACE2 receptor.[9] (Fig-1)

Many proteins are encoded by the ss (+) m-RNA of the n-CoV2019 and SARS COV along with the S1 spike proteins that aid in the attachment of the virion to the cell membrane with interaction to the ACE2 receptor of the host cell $[\mathbf{1 1 , 1 2}]$. Pairwise sequence alignment studies along with BLASTN (Nucleotide BLAST) search of the RBD and partial S1 protein revealed that nCov-2019 is very much similar to the SARS-COV and bat version of the Beta-Coronaviridae [13]. SWISS-PROT docking studies also revealed an increased affinity of the nCov-2019 to the ACE2 receptor than their counterparts of the SARS-COV and bat versions. [13]

SARS-CoV is primarily considered a respiratory pathogen in humans, still the virus has been detected in the brain tissue of infected patients. Autopsy samples from eight patients with SARS-CoV also revealed the presence of the virus in brain samples by immunohistochemistry, electron microscopy, and real-time reverse transcription-PCR[14].In some patients of neurological sequelae, SARS-COV was detected in samples of cerebrospinal fluids using reverse transcriptase-PCR (RT-PCR) during the phase of acute illness. [15] The above results are consistent with well described high propensity of other CoVs to infect CNS and neuronal tissue cells in general. Human CoVs (HCoV)-OC43 and HCoV-229E which primarily cause the common cold, are also detected in the human brain $[\mathbf{1 6}]$. Neuroinvasive propensity of other different Beta-CoVs were also reported such as MERS-CoV [17], porcine and mouse CoV. [18].Recent studies on 214 COVID-19 patient further displayed that about $88 \%$ (78/88) among the severe patients displayed neuronal tissue manifestations including acute cerebrovascular diseases with impaired consciousness. [19] In the light of above facts, chances of the 
possible neuro-invasion by the $\mathrm{nCoV}-2019$ may enlighten a guiding significance for the treatment along with prevention of the SARS-CoV-2-induced respiratory failure in patients with severe symptoms of the disease .

\section{3- ACE-2 receptor expression and Neuro-tropism of SARS-CoV2}

Tissue specific expression of HUMANE ACE2 is also found in nervous tissue (ACE2 [ENSP00000389326]) (TISSUE2.0). In this segment we are reviewing the possible neurovirulence of SARS-CoV-2 and relate it to neurological tissue expression of ACE2 expression in brain (Fig-2). The hallmark of SARS-CoV2 infection is its receptor specificity. Binding of SARS-CoV2 with ACE2Receptor leads to significant drop of ACE2 level, causing a spike in ACE-1 mediated neuroinflammation, neuronal apoptosis, and neurodegeneration, leading towards COVID-19 mediated neurological impairments.[20] Hence, the tissue distribution of receptor generally correlates with the tropism of the virus. In order to determine the neuro-invading potential of SARS-CoV2 the neurological expression of ACE2 receptor has to be reported. Studies have shown that ACE2 receptors are highly expressed in regions like Sub-Fornical organ, Rostral ventrolateral medulla (RVLM), paraventricular nucleus (PVN) and Nucleus of Tractus solitarius (NTS)[21]. n-CoV-19 can enter the brain via twopathways:(1)-haematogenous spread and (2)- retrograde neuronal transport via the olfactory nerve, the former being the more common amongst the two. However, in order to enter the milieu by haematogenous route the virus has to cross the blood brain barrier (BBB). The phylogenetic relationship ensures the structural and infection pathway based similarity between SARS-CoV and SARS-CoV-2[22,23].ACE2 is expressed in human airway epithelia, lung parenchyma, vascular endothelia, kidney cells, small intestine cells and it mediates the entry of SARS-CoV into human host cells [24-26] whereas MERS enter the host cells via Dipeptidyl peptidase 4(DPP4) which is present in lower respiratory tract, kidney, small intestine, liver and the cells of the immune system $[\mathbf{2 7 , 2 8}]$.Besides, SARS-CoV and MERS CoV particles are also found in brain but the exact routes of their entry into CNS still are not reported. Hematogenous or lymphatic routes seems impossible, especially at the early stage of infection whereas some other literatures 
suggest about the invasion of $\mathrm{CoV}$ via peripheral nerve terminals and then they gain access to CNS via a synapse connected route.[29-31].Henceforth considering higher amount of similarity, SARSCoV-2 also possesses a similar neuroinvasive potential which holds an importance for the development of a treatment strategy.[32] Accumulative evidences suggest that glial cells and neurons possess a significant amount of ACE2 receptors which may be a potential target for COVID-19 [11].

ACE2 transforms Angiotensin-II into Angiotensin-(1-7), thus turning the vasoconstrictor into a vasodilator peptide though upregulation of Nitric Oxide Synthase (NOS) activity [33]. It has been found that the presence of SARS-CoV-2 in general circulation enables it to pass into cerebral circulation where the sluggish movement of the blood within the microcirculation may facilitate the interaction between viral spike proteins and ACE2 expressed in the capillary endothelium. Subsequently, viral particles budding from the capillary endothelium may damage the endothelial lining and gain access to the brain [13].

It is important to mention that the movement of COVID-19 to the brain via the cribriform plate close to the olfactory bulb can be an additional pathway to reach brain which can further create hyposmia among the COVID-19 affected patients [13]. Increased ACE 2 receptors in buccal mucosa (more in tongue than gingiva) may also result in symptoms like loss of taste sensation or altered taste sensation[34].Henceforth, a thorough epidemiological study should be performed to establish the neurovirulence effect of SARS-CoV-2 in order to prioritize and individualize the treatment protocols. The presence of ACE2 receptors on the surface of spinal cord neurons supports the cause of acute myelitis as a post inflectional neurological complication $[35,36]$.

\section{4-Immunological interplay may amplify possibilities of SARS-CoV2 mediated neuroinfection and subsequent neurological impairments}

According to medical researchers from different corners of the world suggest that 'Cytokine storm' i.e. overproduction of cytokines is one of a characteristic evidence of Covid-19 infection [37]. 
The astrocytes which form the BBB and the microglia (CNS macrophages) form an integral part of the immuno-surveillance of the brain. Previously neurotropic strains of CoV like MHVA59 have been found to invade the brain via astrocytes. The cytokine cascade that follows potentiates the invasion of the virus into the brain. TNF- $\alpha$, IL-12 (p40-subunit) plays a major role in it apart from IL6, IL-15 \& IL-1b. [38] Consequent pro-inflammatory state is persistent with the neurovirulence of the virus. Accumulative evidences suggest that glial cells and neurons possess a significant amount of ACE2 receptors which may be a potential target for COVID-19.

Studies in children with COVID-19 have shown that GM-CSF levels were significantly higher in the cerebrospinal fluid (CSF) in SARS-CoV2 patients with CNS impairments than in the serum sample of SARS-CoV2 patients with classical respiratory illness [39].Cases of SARS-CoV causing encephalitis in patients and the large homologue sequences it shares with SARS-CoV2 makes it likely that new strain can also pose a threat to the CNS. It has been shown that SARS-CoV can reach the RVLM via post synaptic transfer from mechano and chemo receptors of the lung. [32].

Intranasal inoculation of MERS-CoV into h-DPP4 transgenic mice precipitating paralysis is also reported[40]. A recent retrospective study in China involving covid19 patients with neurological symptoms may be an evidence of the matter. Among 214 COVID-19 patients, 78 had symptoms which can be divided into CNS symptoms: like headache, dizziness, nausea PNS symptoms like hyposmia, paraesthesia and muscle injury which was manifested as increased creatine kinase in those patients. Further investigation revealed that these patients had reduced platelet and lymphocyte counts [19].Besides this functional exhaustion of antiviral lymphocytes also play a role in COVID-19 as SARS-CoV2 increased NKG2A expression in t lymphocytes and Natural Killer (NK)cells.[41] Increased NKG2A expression in cytotoxic-T-lymphocytes (Tc) and NK cells cannot secrete TNF- $\alpha$, Granzyme-b, interferon gamma (IFN- $\gamma$ ), IL2. As immune patrolling of the brain is largely dependent on CD8+T lymphocytes due to the lack of major histocompatibility antigen this may aid the invading power of the virus. [42].Hence, lymphopenia may be a predisposition towards SARS-CoV2 mediated neuroinfection or SARS-CoV2 positive patients at risk of developing neuro-infection. 
Case report of Acute Necrotizing Encephalopathy (ANE) comes upwith an evidence that intracranial cytokine storm may be happened that led to a breakdown of the BBB.Huang et al. reported that COVID-19 provokes the release of inflammatory cytokines including IL-2,IL-6,IL-7,IL-10,TNFalpha and granulocyte colony-stimulating factor and TNF-alpha,IL-6 and C3 of the complement system are the mainfactors in stimulating the immune system[43].Consecutively, these cytokines can drive neuronal hyper-excitability via activation of glutamate receptors and play a role in development of acute seizures.[37].

\section{5- Neurological disorders with a higher risk factor towards developing COVID-19}

Epidemiological Data suggest about the onset of neurological symptoms among the COVID-19 patients. A recent retrospective study in Wuhan, China show a strong correlation between COVID-19 and associated neurological symptom. The neurological symptoms are subdivided into CNS symptoms (headache, dizziness, nausea), PNS Symptoms (hyposmia, paraesthesia) and muscle injury [19]. According to Association of British Neurologists (ABN), numerous risk factors associated with neurological conditions and treatment affect susceptibility to COVID-19 infection. A limited evidence suggests individuals taking Azathioprine, Mycophenolate-mofetil, methotrexate with or without Prednisone, Infliximab, Rituximab, Ocrelizumab are at increased risk of viral infections. Beside this, some neurological conditions require immunotherapies at a regular interval (e.g. monthly infusions of natalizumab for multiple sclerosis) but the frequent attendance may be incompatible with social distance during pandemic time period [44]. Accumulative evidences suggest that some neurological conditions such as-Multiple Sclerosis, Duchene/Becker dystrophy, Congenital muscular dystrophy, Spinal muscular atrophy, Autoimmune Encephalitis, Cerebral vasculitis, Neurosarcoidosis etc show high to moderate amount of risk to develop COVID-19 and some other neurological conditions such as Ocular myasthenia, Glycogen Storage Disorder show lower amount of susceptibility to develop COVID-19.[44]. 


\section{6-Neuro-COVID19-From a clinical point of view}

An early study published from China reported, neurological manifestations may be detected in more than $1 / 3^{\text {rd }}$ of COVID-19 cases and severely affected patients are more likely to present features of neuro-invasion [19]. Neurological consequences of COVID-19 relate to both CNS as well as PNS involvement. CNS involvement has been reported to present in the form headache, dizziness, ataxia, impaired consciousness and seizures; while the observed features of PNS involvement are neuralgia, hyposmia, hypogeusia and hypopsia. Besides, skeletal muscle damage has also been recorded in literature which occasionally may amount to rhabdomyolysis and resultant renal shutdown [19]. With gradual subsidence of the pandemic, post-infectious complications are supposed to draw more clinical attention. We present below a brief summary of reported neurological manifestations of this ailment from the perspective of a clinician.

The tally of CNS features includes some unusual manifestations for a viral infection; one of which is stroke. A monocentric study from Wuhan documented that 5.7\% of neurological manifestations in COVID-19 can be attributed to acute stroke [45]. Ischemic stroke was much more frequent than hemorrhagic type. The increased propensity of this virus to cause stroke, particularly of ischemic type, may be linked to the cytokine storm that it gives rise to. However, it is speculated that the ACE-2 receptor tropism of the viral particle may contribute to rise in blood pressure, particularly affecting intra-cerebral vessels, resulting in cerebral haemorrhages. Apart from cerebro-vascular events, impaired consciousness has been noted in several of the cases so far and the etiological spectrum includes viral meningitis, encephalitis, hypoxic brain damage and infectious toxic encephalopathy. The Wuhan study finds that approximately $7.5 \%$ patients may present impaired consciousness and the underlying reasons may be variable [19]. Acute necrotizing hemorrhagic encephalopathy (ANE) leading to impaired consciousness as an initial manifestation of COVID-19 has been described in a recent report [46]. This particular case consolidates the assumption that CNS involvement is possible even without direct viral invasion. Previous studies have shown ANE to be associated with influenza and many other viral infections but the above mentioned report is the first one to demonstrate a possible association between ANE and COVID-19. According to another report, a 74 year old man 
with past medical history of atrial fibrillation, cardioembolic stroke, Parkinson's disease, chronic obstructive pulmonary disease (COPD) and recent cellulitis came to the emergency room with encephalopathy and subsequently developed fever with cough [47]. Later X-ray thorax revealed multifocal airspace opacities and ground-glass shadows, characteristic of COVID-19. One more recent report describes a 30 years old healthy female presenting tonic-clonic seizure without a history of substance abuse. She complained of dry cough five days prior to admission and later was found positive for SARS-CoV-2 [37]. Seizure is a known after effect of viral encephalitis. Alternatively, cytokine storm which is an important pathological hallmark of COVID-19 may also cause seizure. A striking case report of a 79-year-old patient without a travel history, presenting with syncope along with normal chest radiograph brings to attention another atypical CNS presentation [48]. Therefore it is understood that CNS manifestations in COVID-19, including atypical ones, are not infrequent. Underestimation of the problem is possible, however, owing to scarcity of published literature at this point. A more pressing concern is the CNS symptoms to appear ahead of the typical respiratory symptoms that the virus is known for.

Seen in Wuhan report, PNS involvement of COVID-19 has a predilection towards cranial nerves as evidenced by hyposmia, hypogeusia and hypopsia [19]. While loss of smell sensation has been widely reported so far, consideration is also raised if hyposmia in this ailment reflects olfactory bulb involvement rather than mere peripheral neuropathy. On the same note, hypopsia, if considered a manifestation of optic neuropathy, would also point towards CNS involvement because optic nerve is thought to be an extension of CNS according to classic neuro-anatomy teaching. Neuropathic pain, another symptom described in the Wuhan report under PNS sub-section, was not explored in detail with respect to its distribution. Further elaboration of neuralgia in future studies therefore will be of importance to advance our understanding of its pathogenesis. Additional evidences of PNS involvement, however, can be gathered from two very recent single case reports. One of them is a report from China that describes a 61-year-old woman presenting acute weakness in both the legs and severe fatigue but no flu like symptoms such as fever and cough. Initially she was diagnosed as GBS but after a few days she was positive for Covid-19. Although causality was not claimed by the 
authors, this particular case once more exemplifies that neurological manifestations may precede the so-called respiratory symptoms [49]. A similar case of myelitis has been documented following SARS-CoV-2 infection and appearance of limb weakness was co-incident with the febrile illness pointing towards para-infectious etiopathology [50]. Anatomically speaking, myelitis should be considered as CNS manifestation. However, it has been discussed here in this section keeping in mind the presentation as quadriparesis which often draws differentials from disorders of peripheral neuroaxis. Supposedly, PNS manifestations are less frequently observed thus far, yet they can precede the respiratory symptoms of COVID-19.

The atypical temporal relation between appearance of neurological and pulmonary symptoms is therefore observable in both CNS and PNS manifestations. In situations like these, a neurologist runs the risk of not only getting an inadvertent exposure but also spreading the virus. Horizontal infections are something that the world is worried about at the moment. We believe that some patients presenting to the neurological facility may be the so-called 'silent spreaders' by virtue of their atypical clinical trajectory. Recognizing this important issue at the earliest may help us all to come in grips with the transmission chain.

Since the onset of SARS-CoV-2 outbreak, if there is one pulmonary manifestation that has received maximum focus, it is ARDS. Evidences suggest a significant percentage of ARDS survivors may suffer long-term cognitive impairment [51]. Several factors, including mechanical ventilation, have been observed to cause decline in higher brain functions following ARDS. Acute injury to blood-brain barrier has been implicated as the underlying mechanism for cognitive impairment following ARDS. Effect of such injury may be amplified if there is pre-existing cognitive impairment that corresponds to chronic blood-brain barrier damage. Patients with brain injury, on the other hand, have been found to develop neurogenic pulmonary oedema. Therefore, it is postulated that the so-called brain-lung axis works both ways. The above observations are particularly relevant in the present circumstances given the need for mechanical ventilation in majority of the severely affected COVID-19 patients. As the pandemic continues to unfold, the number of people getting off mechanical ventilation will rise and long-term cognitive outcomes will come into view. It can be anticipated that not only we shall witness 
cognitive decline lasting for months in this group of patients, but also some of them may progress to premature onset of dementia.

Chronic neurology patients are anticipated to pose unique challenge to neurologists amidst this pandemic. Firstly, they are more predisposed than general population to acquire the virus. Secondly, treatment plan needs to be individualized for patients on long term immune suppression because evidence-based guidelines are lacking at present [52]. Therefore, a more patient-tailored approach has to be adapted by the neurologists in present circumstances. Thirdly, in the midst of social distancing teleneurology is going to become extremely relevant in coming days. Rapid acceptance both on part of the physician and patients will be of help.

\section{Conclusion}

In our review we have tried to establish the neurovirulence of SARS-CoV2 beyond its classical manifestations in cardiorespiratory system with critically ill patients being more susceptible to it. The extent of neuroinvasion of the virus depends on its ability to disrupt the BBB, infect neuroglial cells and on the cytokine mediated inflammatory response as a sequela of it. We have hypothesized that higher phylogenetic similarities between SARS-CoV and SARS-CoV-2 and significant homology between the receptor binding domain(RBD), with simultaneous evidences suggesting higher degree of ACE2 expression in Neuronal and neuroglial cells can enhance the virion attachment, augmenting the neurovirulence of SARS-CoV2. We have also analysed the existing case reports and literature on certain coronavirus of the $\beta$-Coronaviridae family which have neuroinvasive potential as reported during earlier outbreaks. Certain case reports along with supported articles have also suggested the development of neurological disorders in patients during the ongoing outbreak of COVID-19. It would also be worthwhile to investigate if phylogeographically distributed, mutational variances of SARS-CoV-2 strains (A, B and C) are associated with modulations in disease spreading and diversity in clinical presentations in neurological spectrum.

Neurological manifestations are being observed in COVID-19 in a considerable number of cases and occasionally they are seen to precede the typical symptoms of fever and cough. Neurologists must 
practice adequate precaution under such circumstances so as to prevent inadvertent exposure; facilitate early diagnosis and isolation; and break the chain of transmission. Chronic neurology patients, particularly those on immune-suppression, would deserve particular attention from therapeutic perspective. It can be assumed that due to scarcity of reporting at the moment, neurological consequence of SARS-CoV-2 is being underestimated. As we make our way through this pandemic a greater number of descriptions are supposed to come up and a clearer view of the subject will be available.

\section{References}

1. Muhammad Adnan Shereen, SulimanKhan, AbeerKazmi, NadiaBashir Rabeea Siddique COVID-19 infection:Origin, transmission, and characteristics of human coronaviruses :Journal of Advance Research https://doi.org/10.1016/j.jare.2020.03.005,March,2020

2. Marco Cascella; Michael Rajnik; Arturo Cuomo; Scott C. Dulebohn; Raffaela Di Napoli. 20 th March,2020Features, Evaluation and Treatment Coronavirus (COVID-19) https://www.ncbi.nlm.nih.gov/books/NBK554776/

3. Kubo H, Yamada YK, Taguchi F Localization of neutralizing epitopes and the receptorbinding site within the amino-terminal 330 amino acids of the murine coronavirus spike protein. J Virol. 1994 Sep; 68(9):5403-10 https://www.ncbi.nlm.nih.gov/pmc/articles/PMC236940/

4. Cheng PK, Wong DA, Tong LK, Ip SM, Lo AC, Lau CS, Yeung EY, Lim WW :Viral shedding patterns of coronavirus in patients with probable severe acute respiratory syndrome,Lancelet May,2004 https://www.ncbi.nlm.nih.gov/pmc/articles/PMC7112423/

5. Fehr AR, Perlman S. Coronaviruses: an overview of their replication and pathogenesis. Methods Mol Biol. 2015;1282:1-23. (Table1) doi:10.1007/978-1-4939-2438-7_1 
6. Raj VS ${ }^{1}$, Mou H, Smits SL, Dekkers DH, Müller MA, Dijkman R, Muth D, Demmers JA, Zaki A, Fouchier RA, Thiel V, Drosten C, Rottier PJ, Osterhaus AD, Bosch BJ, Haagmans BL Dipeptidyl peptidase 4 is a functional receptor for the emerging human coronavirus-EMC doi: 10.1038/nature12005.

7. Unique and conserved features of genome and proteome of SARS-coronavirus, an early splitoff from the coronavirus group 2 lineage. Snijder EJ, Bredenbeek PJ, Dobbe JC, Thiel V, Ziebuhr J, Poon LL, Guan Y, Rozanov M, Spaan WJ, Gorbalenya AE J Mol Biol. 2003 Aug 29; doi:10.1016/S0022-2836(03)00865-9

8. Molecular interactions in the assembly of coronaviruses. de Haan CA, Rottier PJ Adv Virus Res. 2005; 64():165-230. Doi 10.1016/S0065-3527(05)64006-7

9. Roujian Lu, Xiang Zhao, Juan Li, Peihua Niu, Bo Yang, Honglong Wu, Wenling Wang, Hao Song, Baoying Huang, Na Zhu, Yuhai Bi, Xuejun Ma, Faxian Zhan, Liang Wang, Tao Hu, Hon Zhou, Zhenhong Hu, Weimin Zhou, Li Zhao, Jing Chen, Yao Meng, Ji Wang, Yang Lin, Jianying Yuan, Zhihao Xie, Jinmin Ma, William J Liu, Dayan Wang, Wenbo Xu, Edward C Holmes, George F Gao, Guizhen Wu, Weijun Chen,Weifeng Shi, Wenjie Tann Genomic characterisation and epidemiology of 2019 novelcoronavirus: implications for virus origins and receptor binding . Lancet 2020; 395: 565-74. https://doi.org/10.1016/S0140-6736(20)30251-8

10. Peter Forster, Lucy Forster, Colin Renfrew, and Michael Forster Phylogenetic network analysis of SARS-CoV-2 genomes April 8,2020-04-13 https://doi.org/10.1073/pnas.2004999117

11. Palasca, O., Santos, A., Stolte, C., Gorodkin, J., and Jensen, L. J.(2018) TISSUES 2.0: an integrative web resource on mammalian tissue expression. Database 2018, No. bay003

12. Netland, J., Meyerholz, D. K., Moore, S., Cassell, M., and Perlman, S. (2008) Severe acute respiratory syndrome coronavirus infection causes neuronal death in the absence of encephalitis in mice transgenic for human ACE2. J. Virol. 82 (15), 7264-75 
13. Abdul Mannan Baig*AreebaKhaleeq Usman Ali Hira Syeda Evidence of the COVID-19 Virus Targeting the CNS: Tissue Distribution, Host-Virus Interaction, and Proposed Neurotropic Mechanismshttps://pubs.acs.org/doi/10.1021/acschemneuro.0c00122

14. Multiple organ infection and the pathogenesis of SARS. Gu J, Gong E, Zhang B, Zheng J, Gao Z, Zhong Y, Zou W, Zhan J, Wang S, Xie Z, Zhuang H, Wu B, Zhong H, Shao H, Fang W, Gao D, Pei F, Li X, He Z, Xu D, Shi X, Anderson VM, Leong AS J Exp Med. 2005 Aug $1 ; 202(3): 415-24$.

15. Possible central nervous system infection by SARS coronavirus.Lau KK, Yu WC, Chu CM, Lau ST, Sheng B, Yuen KYEmerg Infect Dis. 2004 Feb; 10(2):342-4.

16. Neuroinvasion by human respiratory coronaviruses. Arbour N, Day R, Newcombe J, Talbot PJ J Virol. 2000 Oct; 74(19):8913-21

17. Li K, Wohlford-Lenane C, Perlman S, et al. Middle East respiratory syndrome coronavirus causes multiple organ damage and lethal disease in mice transgenic for human dipeptidyl peptidase 4. J Infect Dis. 2016;213:712-722.

18. Zhou X, Huang F, Xu L, et al. Hepatitis E virus infects neurons and brains. J Infect Dis. 2017;215(8):1197-1206

19. Mao L, Wang M, Chen S, He Q, Chang J, Hong C, Zhou Y, Wang D, Li Y, Jin H, Hu B. Neurological Manifestations of Hospitalized Patients with COVID-19 in Wuhan, China: a retrospective case series study. MedRxiv. https://doi.org/10.1101/2020.02.22.20026500

20. Francesco Chiappelli Towards Neuro-CoViD-19,DOI: 10.6026/97320630016288

21. Luca Steardo Luca Steardo Jr. Robert Zorec Alexei Verkhratsky Neuroinfection may contribute to pathophysiology and clinical manifestations of COVID-19 https://doi.org/10.1111/apha.13473,March,2020

22. Yuan $\mathrm{Y}^{1,2}$, Cao $\mathrm{D}^{3}$, Zhang $\mathrm{Y}^{2,4}$, Ma $\mathrm{J}^{3}$, Qi $\mathrm{J}^{2}$, Wang $\mathrm{Q}^{5}$, Lu $\mathrm{G}^{6}$, Wu $\mathrm{Y}^{7}$, Yan $\mathrm{J}^{5,8,9,10}$, Shi $\mathrm{Y}^{2,8,9,10}$, Zhang $\mathrm{X}^{3,11}$, Gao GF $\mathrm{F}^{1,2,4,8,9,10,12}$ Cryo-EM structures of MERS-CoV and SARS-CoV spike glycoproteins reveal the dynamic receptor binding domains.doi: 10.1038/ncomms 15092 . 
23. Hulswit $\mathrm{RJ}^{1}$, de Haan $\mathrm{CA}^{2}$, Bosch BJ Coronavirus Spike Protein and Tropism Changes.doi: 10.1016/bs.aivir.2016.08.004. Epub 2016

24. M Donoghue, Fang-yin Freda Hsieh, Susan Acton Published in Circulation research 2000 Medicine, Biology A novel angiotensin-converting enzyme-related carboxypeptidase (ACE2) converts angiotensin I to angiotensin1-9DOI:10.1161/01.RES.87.5.e1

25. Harmer $\mathrm{D}^{1}$, Gilbert M, Borman R, Clark KL Quantitative mRNA expression profiling of ACE 2, a novel homologue of angiotensin converting enzyme.DOI:10.1016/s0014-5793(02)036402

26. Hamming $\mathrm{I}^{1}$, Timens W, Bulthuis ML, Lely AT, Navis G, van Goor H Tissue distribution of ACE2 protein, the functional receptor for SARS coronavirus. A first step in understanding SARS pathogenesis.DOI:10.1002/path.1570

27. Mattern $\mathrm{T}^{1}$, Scholz W, Feller AC, Flad HD, Ulmer AJ Expression of CD26 (dipeptidyl peptidase IV) on resting and activated human T-lymphocytes.DOI:10.1111/j.13653083.1991.tb02548.x

28. Boonacker $\mathrm{E}^{1}$, Van Noorden $\mathrm{CJ}$ The multifunctional or moonlighting protein CD26/DPPIV.DOI:10.1078/0171-9335-00302

29. Li YC ${ }^{1}$, Bai WZ, Hirano N, Hayashida T, Hashikawa T Coronavirus infection of rat dorsal root ganglia: ultrastructural characterization of viral replication, transfer, and the early response of satellite cells. DOI:10.1016/j.virusres.2011.12.021

30. Li YC ${ }^{1}$, Bai WZ, Hirano N, Hayashida T, Taniguchi T, Sugita Y, Tohyama K, Hashikawa Neurotropic virus tracing suggests a membranous-coating-mediated mechanism for transsynaptic communication. DOI:10.1002/cne.2317

31. Andries K, Pensaert MB. Immunofluorescence studies on the pathogenesis of hemagglutinating encephalomyelitis virus infection in pigs after oronasal inoculation.https://www.ncbi.nlm.nih.gov/pubmed/6255837

32. Li YC ${ }^{1}$, Bai $\mathrm{WZ}^{2}$, HashikawaTThe neuroinvasive potential of SARS-CoV2 may play a role in the respiratory failure of COVID-19 patients.DOI:10.1002/jmv.25728 
33. $\mathrm{Xu} \mathrm{J}{ }^{1}$, Sriramula $\mathrm{S}^{1}$, Xia $\mathrm{H}^{1}$, Moreno-Walton $\mathrm{L}^{1}$, Culicchia $\mathrm{F}^{1}$, Domenig $\mathrm{O}^{1}$, Poglitsch $\mathrm{M}^{1}$, Lazartigues $\mathrm{E}^{2}$. Clinical Relevance and Role of Neuronal $\mathrm{AT}_{1}$ Receptors in ADAM17Mediated ACE2 Shedding in Neurogenic Hypertension.

DOI:10.1161/CIRCRESAHA.116.310509

34. Hao Xu, Liang Zhong, Jiaxin Deng, Jiakuan Peng, Hongxia Dan, Xin Zeng, Taiwen Li \& Qianming Chen High expression of ACE2 receptor of 2019-nCoV on the epithelial cells of oral mucosa,February 2020 https://www.nature.com/articles/s41368-020-0074-x

35. Nemoto W, Yamagata R, Nakagawasai O, et al. Effect of spinal angiotensin-converting enzyme 2 activation on the formalin-induced nociceptive response in mice. Eur J Pharmacol 2020;872:172950

36. Ogata Y, Nemoto W, Yamagata R, et al. Anti-hypersensitive effect of angiotensin (1-7) on streptozotocin-induced diabetic neuropathic pain in mice. Eur J Pain 2019;23:739-749.

37. Narges Karimi ${ }^{1, *}$, Athena Sharifi Razavi ${ }^{2}$, Nima Rouhani ${ }^{2}$ Frequent Convulsive Seizures in an Adult Patient with COVID-19: A Case Report http://ircmj.com/articles/102828.html

38. Yun Li, Li Fu, Donna M. Gonzales, Ehud Lavi Coronavirus Neurovirulence Correlates with the Ability of the Virus To Induce Proinflammatory Cytokine Signals from Astrocytes and Microglia, Journal of virology, DOI: 10.1128/JVI.78.7.3398-3406.2004

39. Li Y ${ }^{1}$, Li H, Fan R, Wen B, Zhang J, Cao X, Wang C, Song Z, Li S, Li X, Lv X, Qu X, Huang R, Liu W. Coronavirus Infections in the Central Nervous System and Respiratory Tract Show Distinct Features in Hospitalized Children. https://www.ncbi.nlm.nih.gov/pubmed/28103598

40. Guangyu Zhao, Yuting Jiang ,Hongjie Qiu,Tongtong Gao, Yang Zeng,Yan Guo,Hong Yu,Junfeng Li,Zhihua Kou,Lanying Du,Wenjie Tan,Shibo Jiang,Shihui Sun ,Yusen Zhou Multi-Organ Damage in Human Dipeptidyl Peptidase 4 Transgenic Mice Infected with Middle East Respiratory Syndrome-Coronavirus https://doi.org/10.1371/journal.pone.0145561 
41. Meijuan Zheng, Yong Gao, Gang Wang, Guobin Song, Siyu Liu, Dandan Sun, Yuanhong Xu \& Zhigang Tian Functional exhaustion of antiviral lymphocytes in COVID-19 patients https://www.nature.com/articles/s41423-020-0402-2

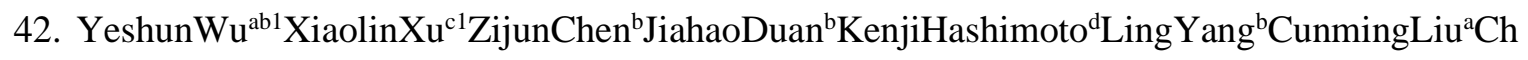
unYang Nervous system involvement after infection with COVID-19 and other coronaviruses,March,2020 https://doi.org/10.1016/j.bbi.2020.03.031

43. Huang C, Wang Y, Li X, Ren L, Zhao J, Hu Y, et al. Clinical features of patients infected with 2019 novel coronavirus in Wuhan, China. Lancet. 2020;395(10223):497-506. doi: 10.1016/S0140-6736(20)30183-5. [PubMed: 31986264].

44. Association of British Neurologists Guidance on COVID-19 for people with neurological conditions, their doctors and carers https://cdn.ymaws.com/www.theabn.org/resource/collection/6750BAE6-4CBC-4DDB-A684116E03BFE634/ABN_Neurology_COVID-19_Guidance_22.3.20.pdf

45. Li, Y., Wang, M., Zhou, Y., Chang, J., Xian, Y., Mao, L. et al., 2020. Acute Cerebrovascular disease following COVID-19: a single centre, retrospective, observational study. The Lancet (Preprint Research Paper).

46. Neo Poyiadji, Gassan Shahin, Daniel Noujaim, Michael Stone, Suresh Patel, Brent Griffith COVID-19-associated Acute Hemorrhagic Necrotizing Encephalopathy: CT and MRI Features https://doi.org/10.1148/radiol.2020201187

47. Asia Filatov, Pamraj Sharma, Fawzi Hindi, Patricio S. Espinosa Neurological Complications of Coronavirus Disease (COVID-19): Encephalopathy doi:10.7759/cureus.7352

48. Tapé $\mathrm{C}^{1}$, Byrd $\mathrm{KM}^{2}$, Aung $\mathrm{S}^{2}$, Lonks $\mathrm{JR}^{2}$, Flanigan $\mathrm{TP}^{2}$, Rybak NR ${ }^{2}$ COVID-19 in a Patient Presenting with Syncope and a Normal Chest X-ray https://www.ncbi.nlm.nih.gov/pubmed/32226962

49. Hua Zhao $\dagger$, Dingding Shen†, Haiyan Zhou†, Jun Liu, *Sheng Chen April 1,2020 GuillainBarré syndrome associated with SARS-CoV-2 infection: causality or coincidence? DOI:https://doi.org/10.1016/S1474-4422(20)30109-5 
50. Zhao, K., Huang, J., Dai, D., Feng, Y., Liu, L., Nie, S., 2020. Acute myelitis after SARSCoV-2 infection: a case report. MedRxiv. doi: https://doi.org/10.1101/2020.03.16.20035105

51. Sasannejad, C., Ely, E.W. \& Lahiri, S. Long-term cognitive impairment after acute respiratory distress syndrome: a review of clinical impact and pathophysiological mechanisms. Crit Care 23, 352 (2019). https://doi.org/10.1186/s13054-019-2626-z

52. Brownlee W, Bourdette D, Broadley S, Killestein J, Ciccarelli O., 2020. Treating multiple sclerosis and neuromyelitis optica spectrum disorder during the COVID-19 pandemic.

Neurology; DOI: 10.1212/WNL.0000000000009507

A
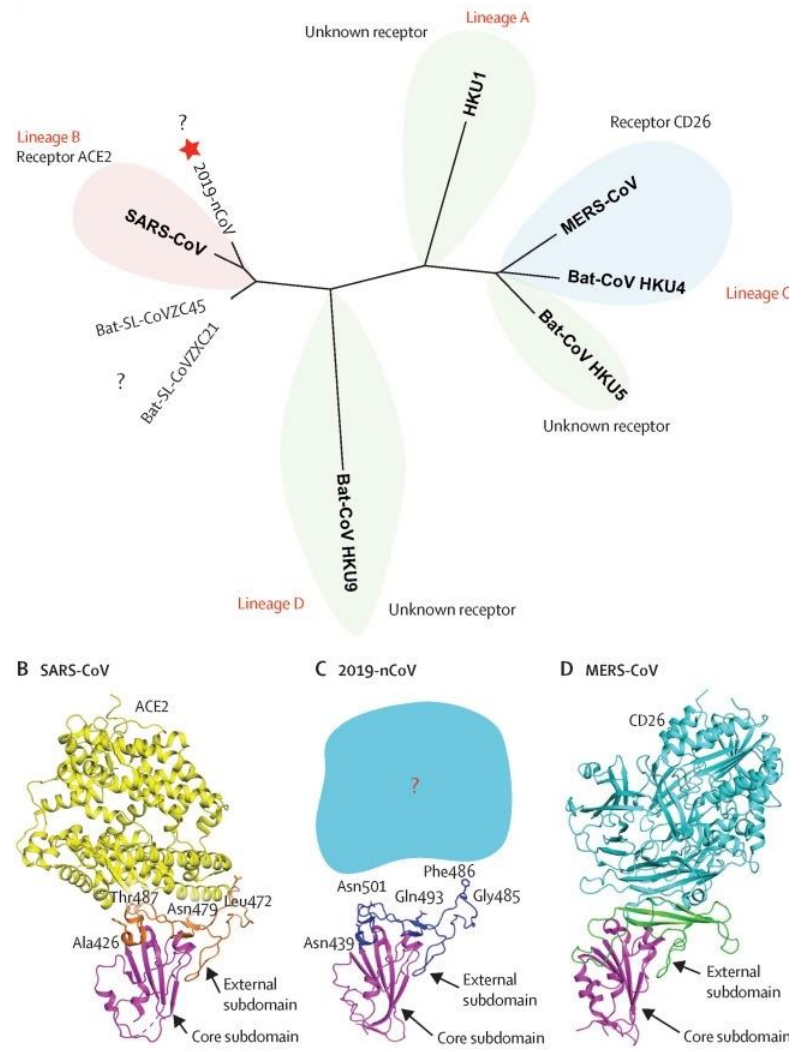

Figure-1 A. Phylogenetic network showing receptor binding domains from various betacoronaviruses. The star denotes $\mathrm{nCoV}-19$ and the question marks means unknown receptor used by the viruses.B,C,D depict structural comparison of the receptor binding domain of SARS$\mathrm{CoV}$,nCoV-19,MERS-CoV binding to their own receptors respectively. Magenta colour represents core domain and the external subdomains of SARS-CoV,nCoV-19,MERS CoV are represented by orange, blue and green respectively [9] 


\section{ACE2 tissues}

\section{ACE2 [ENSP00000389326]}

Angiotensin-converting enzyme homolog; Carboxypeptidase which converts angiotensin I to angiotensin 1-9, a peptide of unknown function, and angiotensin II to angiotensin 1-7, a vasodilator. Also able to hydrolyze apelin- 13 and dynorphin-13 with high efficiency. May be an important regulator of heart function.

Synonyms: ACE2, ACE2p, hACE2, Q9BYF1, Q9BYF1p ...

Linkouts: STRING Pharos UniProt
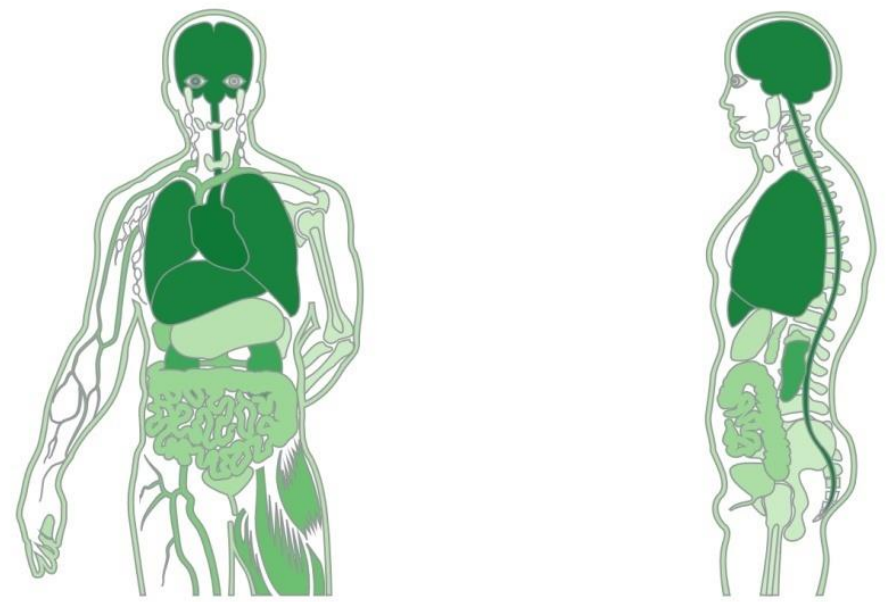

Confidence

$\begin{array}{lllllll}\square & \square & & & & \square\end{array}$

\section{Knowledge}

\begin{tabular}{|c|c|c|c|}
\hline Name & Source & Evidence & Confidence \\
\hline Bile & UniProtKB-RC & CURATED & 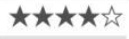 \\
\hline Brain & UniProtKB-RC & CURATED & $\star \star \star \star \star れ$ \\
\hline Heart & UniProtKB-RC & CURATED & 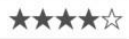 \\
\hline Liver & UniProtKB-RC & CURATED & 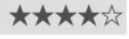 \\
\hline Lung & UniProtKB-RC & CURATED & 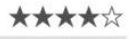 \\
\hline Lymphoma cell & UniProtKB-RC & CURATED & 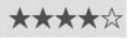 \\
\hline Testis & UniProtKB-RC & CURATED & 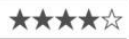 \\
\hline
\end{tabular}

Figure-2 Based on the manually curated knowledge in UniProtKB and via automatic text mining of the biomedical literature ,tissue associations are derived. The confidence of each association is

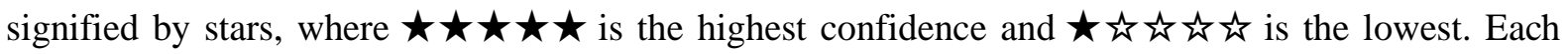
tissue-gene association is represented on a text-mining score, which is proportional to 1) the absolute number of comentionings and 2) the ratio of observed to expected comentionings (i.e. the enrichment). These scores are normalized to z-scores by comparing them to a random background. This is represented by stars, each star corresponding to two standard deviations above the mean of the background distribution.[TISSUE2.0] 
